\title{
Suppression of chaos through reverse period doubling in coupled directly modulated semiconductor lasers
}

\author{
Thomas Kuruvilla ${ }^{1}$, V.M. Nandakumaran \\ International School of Photonics, Cochin University of Science and Technology, Cochin 682022, India ${ }^{2}$ \\ Received 17 June 1998; accepted for publication 13 January 1999 \\ Communicated by A.R. Bishop
}

\begin{abstract}
The effect of coupling on two high frequency modulated semiconductor lasers is numerically studied. The phase diagrams and bifurcation diagrams are drawn. As the coupling constant is increased the system goes from chaotic to periodic behavior through a reverse period doubling sequence. The Lyapunov exponent is calculated to characterize chaotic and periodic regions. (c) 1999 Elsevier Science B.V.
\end{abstract}

PACS: $05.45 ; 42.50 ; 42.55 . \mathrm{b}$

Keywords: Modulated semiconductor lasers; Coupled systems; Chaos; Reverse period doubling; Lyapunov exponents

\section{Introduction}

Coupled nonlinear dynamical systems have been widely studied in the last few years. They exhibit a wide range of interesting phenomena and have found application in a variety of different fields. The dynamics of coupled systems like neural networks [1], chemical oscillators [2], coupled maps [3], Josephson junction [4], etc. have been studied. Coupled laser systems have also been studied [5]. However, many of the earlier studies on coupled laser systems were concerned with the spatial properties of the output radiation rather than their intrinsic dynamical properties. Recently Liu et al. [6] have studied the effect of coupling of two chaotic $\mathrm{CO}_{2}$ lasers with sat-

\footnotetext{
' Permanent address: Department of Physics, Bishop Moore College, Mavelikara 690110. Kerala, India. E-mail: bmc@md3.vsnl. net.in.

2E-mail: photonix@md2.vsnl.net.in.
}

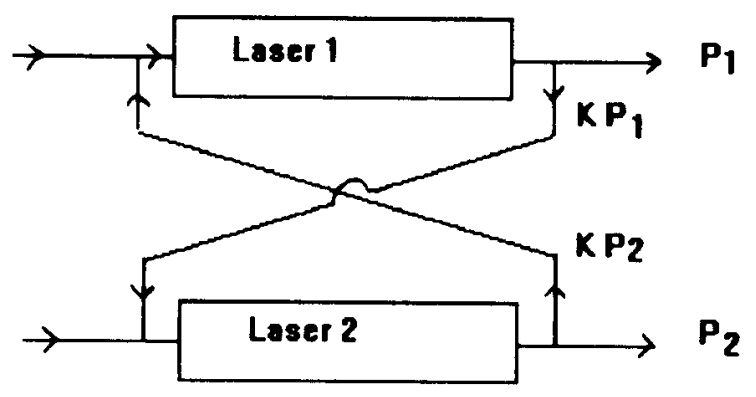

Fig. I. Schematic diagram for coupled lasers.

urable absorbers and Thornburg et al. [7] have studied the chaotic behavior of two coupled single mode $\mathrm{Nd}$-YAG lasers. In this paper we report the results of our numerical studies on two coupled chaotic semiconductor lasers with high frequency current modulation. Semiconductor lasers with high frequency current modulations are important for large capacity information transmission, for ultrafast optical processing and for high speed pulse generation. Such lasers 
have been shown to exhibit chaotic behavior for certain parameter values, both experimentally [8] and theoretically $[9,10]$.

The influence of the nonlinear gain suppression factor [11], spontaneous emission factor [12], Auger combination factor [13], and the effect of noise on such lasers [14] have been studied. Agrawal [11] has shown that the nonlinear gain suppression factor can elimiñate the perioud doubling route to chaos in current modulated semiconductor lasers. However, the chaotic behavior is possible when the gain suppression factor is low.

\section{The model}

The numerical studies on current modulated semiconductor lasers were based on a set of rate equations for the photon density $(S)$ and carrier density $(n)$. The current modulation provides the third degree of freedom necessary for the generation of chaos. Considering the nonlinear gain suppression factor and the effect of noise, the rate equations can be written as [14]

$\frac{\mathrm{d} n}{\mathrm{~d} t}=\frac{l}{e V}-\frac{n}{\tau_{\mathrm{e}}}-A\left(n-n_{0}\right) S+\frac{F_{\mathrm{n}}(t)}{V}$,

$\frac{\mathrm{d} S}{\mathrm{~d} t}=\Gamma A\left(n-n_{0}\right)\left(1-\varepsilon_{\mathrm{NL}} S\right) S-\frac{S}{\tau_{\mathrm{p}}}+\frac{\Gamma \beta n}{\tau_{\mathrm{e}}}+\frac{F_{\mathrm{s}}(t)}{V}$,

where

$I=I_{\mathrm{b}}+I_{\mathrm{m}} \sin \left(2 \pi f_{\mathrm{m}} t\right)$

is the driving current, $e$ is the charge of the electron, $V$ is the active volume, $\tau_{\mathrm{e}}$ and $\tau_{\mathrm{p}}$ are the electron and photon life times respectively, $A$ is the gain constant, $n_{0}$ is the carrier density required for transparency, $\Gamma$ is the confinement factor, $\beta$ is the spontaneous emission factor, $\varepsilon_{\mathrm{NL}}$ is the nonlinear gain reduction occurring with increase in $S, I_{b}$ is the bias current, $I_{m}$ is the modulation current and $f_{\mathrm{m}}$ is the modulation frequency. $F_{\mathrm{s}}(t)$ and $F_{\mathrm{n}}(t)$ are the Langevin noise sources with zero mean that arise respectively from the spontaneous emission and from the discrete nature of carrier generation and recombination.

The photon density and carrier density can further be normalized for numerical purposes by defining $N=$ $n / n_{\mathrm{th}}$ and $P=S / S_{0}$ with $S=\Gamma\left(\tau_{\mathrm{p}} / \tau_{\mathrm{e}}\right) n_{\mathrm{th}}$ where $n_{\mathrm{th}}$ is the threshold carrier density. $n_{\mathrm{th}}=\tau_{\mathrm{e}} I_{\mathrm{th}} / \mathrm{eV}, I_{\mathrm{th}}$ is the threshold current. Correspondingly, neglecting the effect of noise, the rate equations become [11]

$$
\begin{aligned}
& \frac{\mathrm{d} N}{\mathrm{~d} t}=\frac{1}{\tau_{\mathrm{e}}}\left(\frac{I}{I_{\mathrm{th}}}-N-\left(\frac{N-\delta}{1-\delta}\right) P\right), \\
& \frac{\mathrm{d} P}{\mathrm{~d} t}=\frac{1}{\tau_{\mathrm{p}}}\left(\frac{N-\delta}{1-\delta}(1-\epsilon P) P-P+\beta N\right),
\end{aligned}
$$

where $\delta=n_{0} / n_{\mathrm{th}}$ and $\epsilon=\varepsilon_{\mathrm{NL}} S_{0}$ are dimensionless parameters. For certain parameter values the output power shows chaotic behavior [11]. In this paper we have considered the coupling of two such identical chaotic lasers coupled in such a way that a current in milliamperes proportional to the output power of each laser is electronically fed to the other as shown schematically in Fig. 1. The corresponding rate equations can be written as

$$
\begin{aligned}
& \frac{\mathrm{d} N_{1}}{\mathrm{~d} t}=\frac{1}{\tau_{\mathrm{e}}}\left(\frac{I_{1}}{I_{\mathrm{th}}}-N_{1}-\left(\frac{N_{1}-\delta}{1-\delta}\right) P_{1}\right), \\
& \frac{\mathrm{d} P_{1}}{\mathrm{~d} t}=\frac{1}{\tau_{\mathrm{p}}}\left(\frac{N_{1}-\delta}{1-\delta}\left(1-\epsilon P_{1}\right) P_{1}-P_{1}+\beta N_{1}\right), \\
& \frac{\mathrm{d} N_{2}}{\mathrm{~d} t}=\frac{1}{\tau_{\mathrm{e}}}\left(\frac{I_{2}}{I_{\mathrm{th}}}-N_{2}-\left(\frac{N_{2}-\delta}{1-\delta}\right) P_{2}\right), \\
& \frac{\mathrm{d} P_{2}}{\mathrm{~d} t}=\frac{1}{\tau_{\mathrm{p}}}\left(\frac{N_{2}-\delta}{1-\delta}\left(1-\epsilon P_{2}\right) P_{2}-P_{2}+\beta N_{2}\right), \\
& I_{1}=I_{\mathrm{b}}+I_{\mathrm{m}} \sin \left(2 \pi f_{\mathrm{m}} t\right)+K P_{2}, \\
& I_{2}=I_{\mathrm{b}}+I_{\mathrm{m}} \sin \left(2 \pi f_{\mathrm{m}} t\right)+K P_{1} .
\end{aligned}
$$

Here $K$ is the proportionality factor and the subscripts 1 and 2 represent the first and second lasers respectively.

\section{Numerical results}

Eqs. (6)-(11) were studied numerically for various values of the coupling constant $K$ and the results are presented. The numerical integrations were done using a Runge-Kutta fourth order method. The values of parameters used for numerical calculations are given in Table 1. In Fig. 2 we present the phase portrait of laser 1 (power versus carrier density) for various values of the coupling constant $K$. When $K$ is small $(K=2)$ the phase portrait shows chaotic behavior. As $K$ is increased the nature of the orbit changes, at 

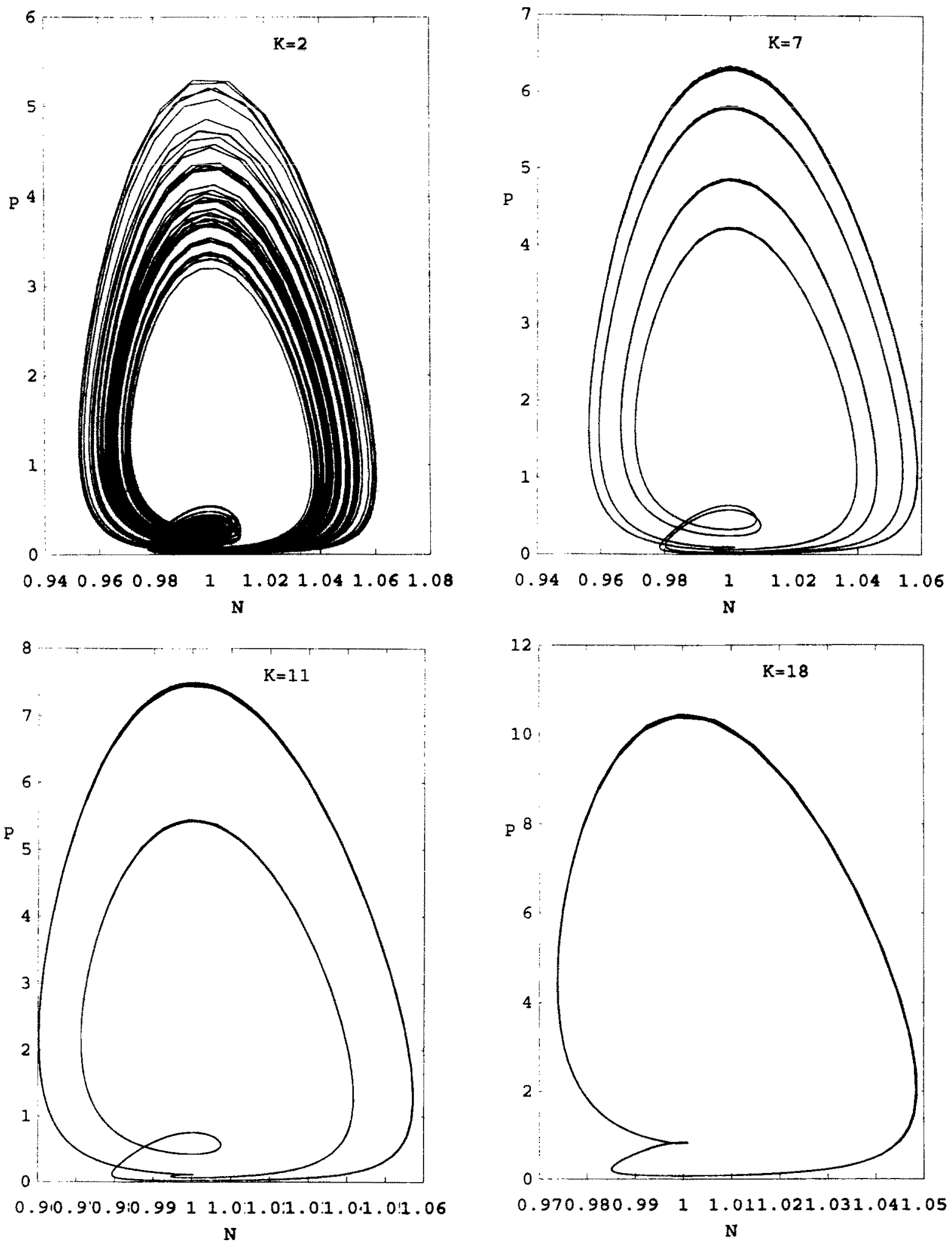

Fig. 2. Phase portrait (power versus carrier density) for laser 1 when the coupling constant $K=2, K=7, K=11$ and $K=18$. 

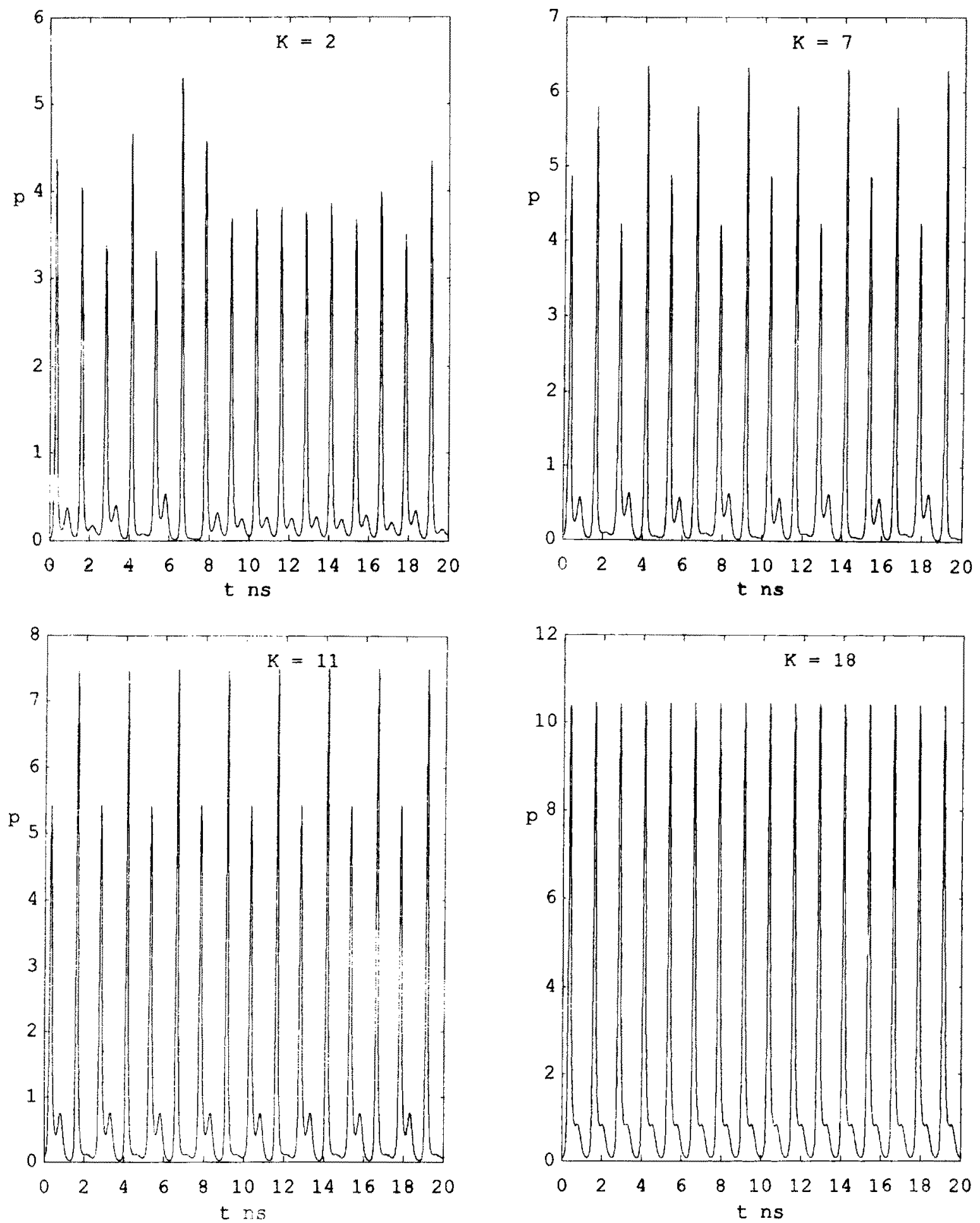

Fig. 3. Pulse train (power versus time) for laser 1 when the coupling constant $K=2$ (chaotic), $K=7$ (period 4). $K=11$ (period 2) and $K=18$ (period 1$)$. 
Table 1

Parameter values used for numerical calculations

\begin{tabular}{ll}
\hline Parameter & Value \\
\hline$\tau_{\mathrm{i}}$, electron life time & $3 \mathrm{~ns}$ \\
$\tau_{\mathrm{p}}$, photon life time & $6 \mathrm{ps}$ \\
$\beta$, spontaneous emission factor & $5 \times 10^{-5}$ \\
$\delta=n_{\mathrm{o}} / n_{\mathrm{h}}$ & 0.692 \\
$\epsilon$, nonlinear gain suppression factor & 0.0001 \\
$f_{\mathrm{m}}$, modulation frequency & $8 \mathrm{Mhz}$ \\
$I_{\mathrm{th}}$, threshold current & $26 \mathrm{~mA}$ \\
$I_{\mathrm{h}}$, bias current & $1.5 I_{\mathrm{h}}$ \\
$I_{\mathrm{m}}$, modulation current & $0.3 I_{\mathrm{h}}$ \\
\hline
\end{tabular}

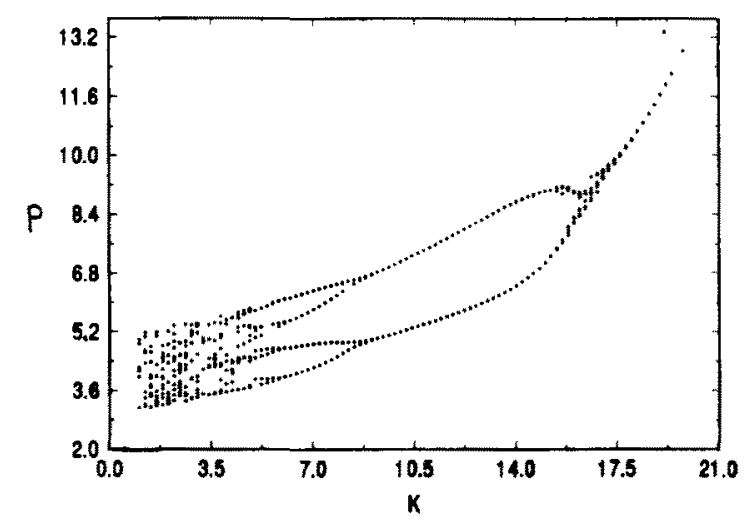

Fig. 4. Bifurcation diagram for the laser.

$K=7$ we see a four-cycle. As $K$ is increased further, the phase diagram exhibits a reverse period doubling bifurcation. For $K=11$ the phase diagram shows a two-cycle and for $K=18$ it shows a one-cycle. This behavior is also seen from Fig. 3 where the power $P$ is plotted against time for the same values of $K$. The power variations are chaotic for $K=2$ while it is periodic, with period four for $K=7$, with period two for $K=11$ and with period one for $K=18$. The second laser also showed similar behavior.

Fig. 4 shows the bifurcation diagram for the laser. It is seen from the bifurcation diagram that as the coupling constant $K$ is increased the system goes from chaotic to steady state (one cycle) through a sequence of reverse period doubling. This transition to periodic behavior is also evident from the values of the Lyapunov characteristic exponent (LCE) calculated from numerically obtained output power time series. The Wolf et al. algorithm [15] is used for the calculation of the LCE. The LCE calculated for different $K$ values are given in Table 2. Again it is seen that for $K=2$
Table 2

Lyapunov exponents calculated from numerically obtained output power time series with the picosecond as the unit of time

\begin{aligned} & \hline$K$ Lyapunov exponent \\ & \hline 2$+1.928 \times 10^{-4} \\ & 7-1.864 \times 10^{-4} \\ & 11-3.662 \times 10^{-4} \\ & 18-3.682 \times 10^{-4} \\ &$\hline\end{aligned}

the LCE is positive showing chaotic behavior while it is negative for higher values of $K$, showing regular behavior.

\section{Conclusion}

Our numerical studies show that the coupling of two lasers as described here has the effect of suppressing chaos of the lasers. By increasing the coupling constant $K$ the system is made to undergo a cascade of inverse period doubling bifurcations. By suitably choosing the $K$ values one can bring the output to any desired cycle.

\section{Acknowledgement}

One of the authors (TK) wishes to thank UGC, New Delhi for a minor research grant and VMN acknowledges the financial support through a minor research project under the UGC scheme of an unassigned grant.

\section{References}

[1] M. Kavato, R. Suzuki, J. Theor. Bio. 86 (1980) 547.

[2] K. Bar-Eli, Physica D 14 (1985) 242.

[3] K. Kaneko, Prog. Theor. Phys. 69 (1983) 1806.

[4] S. Watanabe, S.H. Strogatz, H.S.J. Vander Zant, T.P. Orlando, Phys. Rev. Lett. 75 (1995) 45.

[5] H. Winful, R.K. DeFreez, in: Diode Laser Arrays, eds. D. Botez, D.R. Serifres (Cambridge Univ. Press, Cambridge, 1994).

16] Y. Liu, J.R.R. Leit, Phys. Lett. A 191 (1994) 134.

[7] K.S. Thornburg Jr., M. Moller, R. Roy, Phys. Rev. E 55 (1997) 3865.

[8] H.G. Winful, Y.C. Chen, J.M. Liu, Appl. Phys. Lett. 48 (1986) 616.

[9] M. Tang, S. Wang, Appl. Phys. Lett. 48 (1986) 900.

[10] Y. Hori, H. Serisava, H. Sato, J. Opt. Soc. Am. B 5 (1988) 1128. 
[11] G.P. Agrawal, Appl. Phys. Lett. 49 (1986) 1013.

[12] M. Tang, S. Wang, Appl. Phys. Lett. 47 (1985) 208.

(13) M. Tang, S. Wang, Appl. Phys. Lett. 50 (1987) 1861.
[14] Y.H. Kao, H.T. Lin, IEEE J. Quant. Electr. 29 (1993) 1617.

[15] A. Wolf, J.B. Swift, H.I. Swinney, V. Vastano, Physica D 16 (1985) 285. 